\title{
A fuzzy-based approach to evaluate multi-objective optimization for resource allocation in cloud
}

\author{
Bela Shrimali $^{1^{*}}$, Harshad Bhadka ${ }^{2}$ and Hiren Patel $^{3}$ \\ Research Scholar, Department of Computer Science, C.U.Shah University, Wadhwan, Surendranagar, Gujarat, \\ India $^{1}$ \\ Dean, Department of Computer Science, C.U.Shah University, Wadhwan, Surendranagar, Gujarat, India ${ }^{2}$ \\ Professor, Department of Computer Science, LDRP Institute of Technology and Research, Gandhinagar, Gujarat, \\ India $^{3}$

\section{(O2018 ACCENTS}

\begin{abstract}
Effective resource allocation can be used to achieve two important parameters in Cloud viz. energy efficiency and data center performance. Multi-objective optimization is one of the techniques to address the issue of resource allocation with the multiple objectives. In this research, we aim to address the issue of resource allocation through a weighted sum based multi-objective optimization technique. In weighted sum method, coefficient is attached with each objective as a user's preferences to decide a priority of objective. Genetic algorithm and fuzzy logic are the identified methods to calculate the co-efficient to generate Pareto optimal solutions. In this paper, we use fuzzy logic to generate the random value of objectives' co-efficient. The proposed fuzzy-based computing is implemented and experimental results show the proposed scheme efficiently generates a random coefficient that assigns priority by considering characteristics of host. Results depict the average improvement in performance by $25.7 \%$ in power and $3.67 \%$ service level agreement (SLA) violations over the period of 24 hours. Further, it demonstrates that the weight generated gives Pareto optimum solution that points to strict Pareto curve.
\end{abstract}

\section{Keywords}

Fuzzy logic, Co-efficient, Weighted sum method, Preferences/priority.

\section{Introduction}

Intensive use of computing technologies by industries, scientific applications and end users makes Cloud computing a very popular paradigm. The success of existing Cloud infrastructures like Amazon's elastic compute Cloud and GoogleCloud platforms inspire the institutions to move to the private Cloud. Cloud infrastructure comprises of a data center with large pool of resources and the efficient use of available resource increases the throughput of Cloud. Yet, performance, availability, security, energy consumption, cost effects/revenue generation and efficient resource allocations are few of the challenges attached with Cloud for its overall adoption.

The quality of service (QoS) in Cloud environment depends upon the performance of physical host/server in use.

\footnotetext{
*Author for correspondence
}

Cloud infrastructure that contains a large number of servers, disks, network devices make it possible to handle millions of requests from users around the globe. However, the consumers need to pay for usage to the service providers. On the service provider's side, maintenance and management of this large-scale data centers need to be emphasized for revenue generation.

Due to enormous computing requirement worldwide, over the past years, the amount of data centers has increased considerably. This has led to an issue of energy consumption by these data centers and subsequently affecting environment and financial impacts. For instance, in Amazon's data centers, it is identified and reported that [1] i) Expenses related to the cost and operation of the servers is $53 \%$ of total budget. ii) Energy-related costs is about $42 \%$ of total budget that includes both, direct energy consumption of $19 \%$ by servers and power used in cooling the infrastructure about $23 \%$. It has been also mentioned in Gartner Report, that IT industry contributes total $2 \% \mathrm{CO} 2$ emissions in environment. US EPA report in 
2007 has also mentioned that $1.5 \%$ of total US power consumption is used by data centers and costs $\$ 4.5$ billion. Thus, it is very significant for service providers to think upon the two major affecting factors viz. performance and power consumption in Cloud.

Resources in Cloud are provisioned in virtual form rather than actual physical hardware. Virtualization is the key technology to provide resources in the form of virtual machine (VM) as an instance of physical machine. The process of allocating available resources to the different processes without compromising the system's integrity is called resource allocation. Starvation and deadlock are the main issues that can be resolved with proper resource allocation mechanism. First in first out (FIFO), shortest-job-first (SJF), round robin (time-slice scheduling), priority-based preemptive scheduling are the few resource scheduling policies. In Cloud, issues of maintenance, management and extendibility of resources can be handled by efficient resource allocation.

Inefficient resource allocation may result into several issues such as more power consumption, unbalanced utilization of available resources and service level agreement (SLA) violations. There are many strategies and methods [2] like round robin, load balancing, load sharing and stripping used by popular cloud platforms OpenNebula [3] and Eucalyptus[3]. This resource allocation in the form of VM allocation is further categorized based on the different parameters like energy, network, SLA, data awareness and performance [4]. Efficient resource allocation policies could be used to address these factors. The process of efficient resource allocation may address this issue of performance and energy consumption in cloud data center. Energy consumption and performance of the data center are two of the parameter that effect to the revenue. Hence, these parameters need to be considered for cost effective resource allocation in cloud.

Energy-efficient allocation with performance maintenance in the Cloud is yet a challenging issue, as both parameters conflict each other. One of the challenges of energy-efficient and performance oriented scheduling algorithms is the trade-off between energy consumption and performance. Hence, in this research, we aim to address a fuzzy based method that prioritizes the objectives based on the host's current conditions/characteristics.
Comparative study on performance and energy efficient allocation techniques carried out in [4] conclude that there is a trade-off between performance and energy consumption. Objectives with the trade-off can be optimized simultaneously with the method of mathematics called as multiobjective optimization (MOO) approach. This method will generate set of non-dominated Pareto optimal solutions. Rather, to obtain single optimal solution like single objective optimization. There are four basic approaches of MOO viz. methods with apriori articulation of preferences, methods with a posteriori articulation of preferences, methods with no articulation of preferences and progressive articulation of preferences or interactive method [5]. These methods are analyzed and compared based on identified parameters. Their comparisons are discussed in detail in our previous work [6]. Based on the analytical study made on different techniques, we identified weighted sum method of apriori articulation of preferences for MOO suitable for our method, where a prior weightages are given to different objectives by service provider. This weightages are actually a user's priorities to objectives. In weighted sum method, if the weights are not identified accurately, it may not generate the optimized solutions. In case of MOO objectives are non-dominated and hence, single optimized solution is not appropriate. Fuzzy logic and genetic algorithms are identified approaches to generate the multiple optimized solutions. Different methods for both of these are discussed in related work. Here, we analyzed, discussed and compared both approaches as shown in Table 1.

From the Table 1, we can conclude that fuzzy approach is most suitable to generate a weight/coefficient for our proposed weight based MOO based resource allocation problem and hence, in this paper, we have used fuzzy method to generate a weight/coefficient for every objective of multiobjective equation.

The rest of the paper is organized as follows. Section 2 describes related work. Section 3 describes analytical model and problem statement of MOO allocation policy, followed by proposed algorithm in section 4. Conclusion and future work are depicted in section 5. The list of references used in the paper in section 6 .

\section{Related work}

In this section, we have reviewed current research on MOO and fuzzy-based resource allocation with 
Shrimali et al.

different methods of evaluating a coefficient of multiobjective optimization. Particularly, for weighted sum method of multi-objective optimization, to provide a weight, few methods are identified and briefly discussed [5, 7-9]. Marler and Arora [5] have focused and discussed different methods to generate a weight like ranking methods, categorization methods, rating methods, ratio questioning or paired comparison method, eigenvalue method of determining weights and method of fuzzy logic.

Table 1 Comparison of fuzzy logic method with genetic algorithm for weighted sum approach

\begin{tabular}{|c|c|c|c|}
\hline Approach/method & Characteristics & Advantage & Disadvantage \\
\hline Fuzzy logic & $\begin{array}{l}\text { Based on the fuzzy rules and fuzzy } \\
\text { logic, weights are calculated that } \\
\text { generate optimal solutions after } \\
\text { number of iterations. }\end{array}$ & $\begin{array}{l}\text { A straight forward method that } \\
\text { uses host's characteristics to } \\
\text { generate appropriate weight. }\end{array}$ & $\begin{array}{l}\text { Appropriate and clear fuzzy } \\
\text { rules are required to generate } \\
\text { optimal Pareto front for non- } \\
\text { convex surface. }\end{array}$ \\
\hline Genetic algorithm & $\begin{array}{l}\text { Optimal solutions are generated } \\
\text { based on previous solution using } \\
\text { the calculation of fitness function. }\end{array}$ & $\begin{array}{l}\text { Straight forward } \\
\text { implementation. Since a single } \\
\text { objective is used in fitness } \\
\text { assignment, a single objective } \\
\text { GA can be used with minimum } \\
\text { modifications. }\end{array}$ & $\begin{array}{l}\text { Pareto optimal solutions } \\
\text { needs to be analysed and } \\
\text { investigated when the true } \\
\text { Pareto front is non-convex. } \\
\text { So, multi- objective GA } \\
\text { based on the weighted sum } \\
\text { approach have difficulty in } \\
\text { finding solutions } \\
\text { homogeneously distributed } \\
\text { over a non-convex trade-off } \\
\text { surface. }\end{array}$ \\
\hline
\end{tabular}

They have shown the advantages and limitations of weighted sum approach. To generate optimum solutions in MOO, genetic algorithms have been introduced and discussed for different method of MOO in [7]. They have also investigated that the weighted sum approach of multi-objective GA, has difficulty in finding optimum solutions that are uniformly distributed over a non-convex trade-off surface. Rao and Roy [8] have introduced fuzzy based approach of assigning weights to objectives in multi-criteria decision making problems. But, proper ordering of objective functions based on the preferences was prerequisite in their approach. In this paper, we have considered many factors effecting host to decide the preferences of objectives. Also, based on this factors weight is generated.

Masoumzadeh et al. in [9] have used fuzzy based approach for energy and performance efficient dynamic VM consolidation. However, they used fuzzy approach to build intelligent schema for threshold calculation. Xu et al. [10] have used fuzzylogic-based control system for efficient resource allocation. They have used fuzzy approach to identify the mapping of application workload and resources. However, they have focused on resource cost and application's QoS.

Panchal et al. [11] have used entropy based method to evaluate co-efficient/weight attached with each identified objectives.

142
In their proposed work, they have used the ideal and negative ideal solution. However, it depends on the difference between solutions.

In this paper, we have focused on MOO with fuzzy approach to make more efficient resource allocation.

\section{Our proposal}

In this section, we describe the fuzzy-based approach to generate the coefficient for evaluating the MOO expression. Consider a Cloud comprising of a large scale of data center consisting of homogeneous physical nodes. Request for resources is mapped to the different nodes based on the resource availability in addition to the consideration of parameters like resource utilization, performance and energy consumption.

For our method, we have used MOO technique to satisfy the performance and energy consumption need. The proposed MOO technique requires the weightage of objectives.

And hence, it is significant to identify a technique that generates the weight efficiently and further generates a Pareto front.

\subsection{System model}

Requests from the end users are sent to Cloud data centers. Numerous nodes collectively create data 
center. Every node has a component either node controller (NC) or cluster controller (CC). The overall functionality, working and calculation carried out by these components are discussed in our previous work [6]. This work is our extended research for using fuzzy to generate random weightage. Overall system architecture is discussed in our previous work [6]. Fuzzy co-efficient will be calculated by CC.

\subsection{Problem statement}

Proper selection of a weight to illustrate the decision maker's/ service provider's preferences is identified as an important problem. It is very difficult to precisely and accurately select these weights for the equal important objectives. Hence, a single solution is not optimal for the non-dominated objectives. A proper weight component will generate efficient or Pareto optimum solutions for the multi-objective problem.

\subsection{Proposed method}

Through this research, we aim to provide optimal resource allocation option from all possible solutions. Optimal resource allocation policy (ORAP) can be generated in terms of a pair comprising of (i) Request (for a resource) and (ii) Target host (on which the request can be mapped). To compute such pair, two parameters must be included viz. (a) SLA violation and (b) energy consumption. We propose the separate functions for computing these two parameters, $f$ energy $(x, y)$ and $f$ perfo $(x, y)$, where $x$ and $y$ are the configuration specifications of request and host, respectively. We have included here the calculation of this function for better understanding in flow of work. Beloglazov et al. [12] defines the terminology illustrated in Equation1-3. SLA violation (SLAV) time per active node (SLATAN) [12] (where node experience maximum utilization) (Equation 1).

1. Performance degradation due to migration (PDM) [12] of VM (Equation 2).

SLATAN $=1 / \mathrm{N} \sum_{\mathrm{i}=1}^{\mathrm{n}} \mathrm{T}_{\mathrm{Si}} / \mathrm{T}_{\mathrm{ai}}$

$P D M=1 / M \sum_{j=1}^{M} C_{d j} / C_{r j}$

From both of the above, SLAV [12] is defined by equation (3):

$\mathrm{SLAV}=\mathrm{SLATAN} \times \mathrm{PDM}$
Host Utilization $\mathrm{U} \mathrm{H}$ [11] is defined as

$$
\mathrm{U}_{\mathrm{H}}=\sum_{i=1}^{n}(U v m i * C v m i) / \mathrm{C}_{\mathrm{H}}
$$

$\mathrm{C}_{\mathrm{H}}=$ number of core $\times$ individual

core capacity

Energy consumption by host E H [13] is defined as $E_{H}=\left(\mathrm{P}_{\max }-\mathrm{P}_{\min }\right) \times \mathrm{U}_{\mathrm{H}}+\mathrm{P}_{\min }$

As we said earlier, optimal resource allocation depends on $\mathrm{f}_{\text {energy }}(\mathrm{x}, \mathrm{y})$ and $\mathrm{f}_{\text {perfo }}(\mathrm{x}, \mathrm{y})$, we may derive following equation 7 :

$\mathrm{Cal}(\mathrm{r}, \mathrm{h})=\lambda \times \mathrm{f}$ energy

$(\mathrm{x}, \mathrm{y})+(1-\lambda) \times \mathrm{f}$ perfo $(\mathrm{x}, \mathrm{y})$

Where, $\lambda=$ Weightage/service provider's preference.

In our MOO based resource allocation technique viz. ORAP, the value of weight $\lambda$ affects the quality of resource allocation. As discussed in the related Work section, normally researchers consider the random value of $\lambda$ which often results into inefficient resource allocation, and hence, we believe that the value of the weight $\lambda$ should be determined using fuzzy logic for efficient resource allocation. As discussed in later part of this paper, the experimentation results support the claim made here.

Through this research, we contribute in the direction of fuzzy based calculation of $\lambda$. Further, based on various characteristics of host (such as utilization, energy consumption, SLA violation), we give the preference by giving weightage to the objective functions mention in Equation 3.

3.3.1Fuzzy logic system

Fuzzy based system consists four basic functions as shown in Figure 1. The fuzzification function takes crisp input values and mapped it to fuzzy values using membership function. The knowledge base includes a database which contains the membership functions and a rule base that specify the fuzzy rules. The fuzzy inference engine takes the fuzzy inputs and generates the fuzzy output based on the fuzzy rules stored in a rule base. The defuzzification function aggregates the fuzzy output and converts it to crisp output. In this way, $\lambda$ is generated based on the different characteristics of host. 


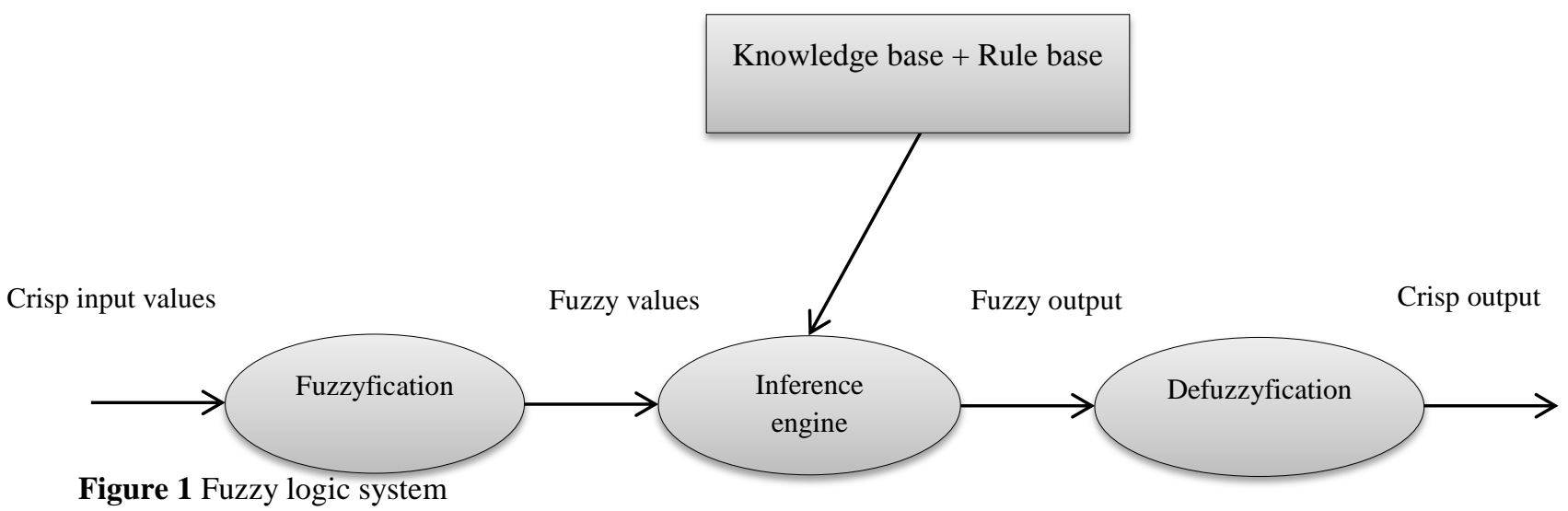

\subsubsection{Crisp input}

These different characteristics of host are input to fuzzy system. They are identified as linguistic variables. Identified linguistic variables of the system are as follows:

- Total number of time host in active state.

- Number of VM migrated.

- Number of time host is experiencing full utilization.

- Utilization of host.

- Power consumption of host.

- SLA violations of host.

From the identified linguistic variables, factors like number of VM migrated, total number of time host in active state and number of time host identified full utilization are the factors that affect the SLA agreement and are the reasons of SLA violation. By considering this, we come up with three linguistic variables which may, in turn, determine others. Final linguistic variables are as follows:

- SLA violations

- Power consumption

- Utilization of host. 25

3.3.3Fuzzy rules

Fuzzy rules are applied to input values of (i) utilization, (ii) power consumption and (iii) SLA violations. Range is defined for the three factors to categorize them. The value of co-efficient $\lambda$ is in the range [0-1]. Fuzzy input of linguistic variables is shown in Table 2.

Table 2 Fuzzy input of linguistic variables

\begin{tabular}{llll}
\hline Input & \multicolumn{3}{l}{ Categorization based on input value } \\
\hline $\begin{array}{l}\text { Utilization of } \\
\text { host }\end{array}$ & Over & Under & Moderate \\
$\begin{array}{l}\text { Energy } \\
\text { consumption }\end{array}$ & Minimum & Maximum & Average \\
SLA & More & Less & \\
Violation & & & \\
\hline
\end{tabular}

Fuzzy rules are as follows:

1. If utilization of host is over, energy consumption is maximum and SLA violations are more, coefficient $\lambda$ is large.

2. If utilization of host is under, energy consumption is average and SLA violations are more, coefficient $\lambda$ is large.

3. If utilization of host is moderate, energy consumption is average and SLA violations are more, co-efficient $\lambda$ is large.

4. If utilization of host is moderate, energy consumption is average and SLA violations are less, co-efficient $\lambda$ is large.

5. If utilization of host is moderate, energy consumption is maximum and SLA violations are more, co-efficient $\lambda$ is small.

6. If utilization of host is moderate, energy consumption is maximum and SLA violations are less, co-efficient $\lambda$ is small.

Fuzzy matrices for the defined rules are as in Table 3 and Table 4.

Table 3 The values of co-efficient $\lambda$ under the circumstances of more SLA violations

\begin{tabular}{lll}
\hline & Mower \\
Consumption & & Average \\
\hline Ovilization & & \\
\hline Under & Large & - \\
Moderate & - & Large \\
\hline
\end{tabular}

Table 4 The values of co-efficient $\lambda$ under the circumstances of less SLA violations

\begin{tabular}{lll}
\multicolumn{1}{c}{ Power } \\
Consumption \\
Utilization
\end{tabular}


3.3.4Membership function

For our work, we have used triangular method to map the values. For each value of linguistic variable $\mathrm{x}$, $\mu \mathrm{A}: \mathrm{x} \rightarrow[0-1]$ will be calculated as shown in equation 8

$$
\mu_{A}(x)=\left\{\begin{array}{l}
0, \quad x<=a, \\
x-a / m-a, \quad \text { if } a<=x<=m \\
b-x / b-m, \text { if } m<=x<=b \\
0, \quad x>=b
\end{array}\right.
$$

where, $\mathrm{a}$ and $\mathrm{b}$ are lower and upper values respectively for each linguistic variables, $m$ is the mean of $a$ and $b$.

There are two types of inference method viz. direct and indirect. Mamdani [14] and Sugeno [15] are the most popular method of direct type. We have selected Mamdani method for inference.

\subsubsection{Defuzzification}

For our work, we have used the Center of Gravity method for defuzzification. This method [15] works as shown in Equation 9.

$\mathrm{U}=\int_{\min }^{\max } u \mu(\mathrm{u}) \mathrm{du} / \int_{\min }^{\max } \mu(\mathrm{u}) \mathrm{du}$

Where,

$\mathrm{U}=$ Result of defuzzification,

$\min =$ lower limit for defuzzification,

$\max =$ Upper limit for defuzzification,

$\mu=$ Membership function.

\subsection{Proposed algorithm}

Algorithm for the calculation of the co-efficient $\lambda$ is as follow:

- Input: Crisp values of linguistic variables. 10

- Output: A fuzzy value of co-efficient $\lambda$.

\footnotetext{
Algorithm 1 Calculation of the co-efficient $\lambda$ (Host H)

1: Initialization of linguistic variables SLA violation as $\mathrm{s}$, energy consumption e and utilization $\mathrm{u}$.

2: Initialize lower_limit a, upper_limit b, m such that $\mathrm{a} \leq \mathrm{m} \leq \mathrm{b}$ for each linguistic variable.

3: for each $\mathrm{H}$ in hostList do do

4: read the values of $\mathrm{s}, \mathrm{e}, \mathrm{u}$;

5: for each linguistic variable $\mathrm{x}$ do
}

6: calculate $\mu$ (x) using triangular membership function using 8 .

7: for each rule in rule set do

8: Aggregate the conclusion of each rule.

9: end for

10: end for

11: end for

12: Apply defuzzification using center of gravity method

13: Print co-efficient $\lambda$

Outcome: Fuzzy value of $\lambda$.

3.5Calculation of co-efficient: analysis

For each host available in hostlist of size $n$, the algorithm evaluates value of fuzzy value $\mathrm{x}$ for each linguistic variable $\mathrm{m}$ and evaluate aggregation of result of each rule set $r$. Hence, the total time to evalute co-efficient $\lambda$ is $\mathrm{O}(\mathrm{mnr})$.

\section{Experimental evaluation}

In this section, initially we start with sample evaluation, in which we have taken different values of host viz. utilization, SLAV and energy consumption. The evaluation scenario describes how these values are affecting in generation of weightage. Further, we demonstrate the simulation scenario (Example Table 5) with the help of experimental results.

\subsection{Sample evaluation}

To perform sample evaluation to generate weightage $\lambda$, we consider 10 hosts with different values of utilization, energy consumption and SLAV as mentioned in Table 5. The table depicts effect of different values of three parameters used in our proposed algorithm for each available. From the Table 5, we can see that as describe in fuzzy rule set, $\lambda$ is randomly generated based on the different values of utilization, energy consumption and SLAV. This fuzzy based $\lambda$ is generated as per host characteristics. The table shows that host having more SLAV, will get more preferences to performance in objective function. This fuzzy based $\lambda$ will iteratively generate Pareto optimal solutions and creates Pareto front.

Table 5 Performance evaluation

\begin{tabular}{lllllll}
\hline Tuple \# & Host_Id & Utilization & $\begin{array}{l}\text { Energy } \\
\text { Consumption }\end{array}$ & $\begin{array}{l}\text { Performance } \\
\text { (SLAV) }\end{array}$ & $\boldsymbol{\Lambda}$ & $\mathbf{1 - \Lambda}$ \\
\hline 1 & H1 & 0.00058 & 0.03355 & 0.000014 & 0.7 & 0.3 \\
2 & H2 & 0.00065 & 0.0117 & 0.05777 & 0.8 & 0.2 \\
3 & H3 & 0.00059 & 0.003 & 0.000057 & 0.4 & 0.6 \\
4 & H4 & 0.00054 & 0.03795 & 0.0000347 & 0.8 & 0.2 \\
5 & H5 & 0.00078 & 0.0143 & 0.0000138 & 0.3 & 0.7 \\
\hline
\end{tabular}


Shrimali et al.

\begin{tabular}{lllllll}
\hline Tuple \# & Host_Id & Utilization & $\begin{array}{l}\text { Energy } \\
\text { Consumption }\end{array}$ & $\begin{array}{l}\text { Performance } \\
\text { (SLAV) }\end{array}$ & $\boldsymbol{\Lambda}$ & $\mathbf{1 - \Lambda}$ \\
\hline 6 & H6 & 0.0005 & 0.0038 & 0.000138 & 0.6 & 0.4 \\
7 & H7 & 0.00067 & 0.06395 & 0.0000465 & 0.6 & 0.4 \\
8 & H8 & 0.00065 & 0.0247 & 0.000018 & 0.6 & 0.4 \\
9 & H9 & 0.0007 & 0.007 & 0.000186 & 0.4 & 0.6 \\
10 & H10 & 0.00062 & -0.0107 & 0.000353 & 0.5 & 0.5 \\
\hline
\end{tabular}

4.2Experimental results

We have simulated our algorithm to apply fuzzy based approach to evaluate MOO for resource allocation. Simulation approach is used to perform the experiments iteratively under an analogous environment. Thus, allocation policies can be compared effectively. The CloudSim [16] has been chosen for simulation as it allows the demonstration of virtualized environments with on-demand resource provisioning and management. In our simulation, we have used two types of power host viz. PowerModelSpecPowerHp-

ProLiantM1110G4Xeon3040 and PowerModelSpecPowerHpProLiantMl110G5Xeon30 75. Also, to use workload traces collected from a real system, PlanetLab[17] workload is used, that consists of different readings of CPU utilization collected at interval of 5 minute of VMs of different host scattered around the world. From the collection of PlanetLab workload, we have used workload taken during march-2011 for our simulation. In our experiments, we have VMs considered to be a resource request type, and the VM characteristics were considered to be the attributes, which include the processing capacity(in MIPS), bandwidth (BW)(Mbps), VM size(GB). PEs denotes processing elements. SLATAH denotes the SLA violation time per active host.

The configuration of physical machines and VMs are as shown in Table 6 and Table 7 respectively.

Table 6 Configuration of physical machines

\begin{tabular}{llllll}
\hline HOST TYPES & $\begin{array}{l}\text { HOST } \\
\text { MIPS }\end{array}$ & $\begin{array}{l}\text { HOST } \\
\text { PES }\end{array}$ & $\begin{array}{l}\text { HOST } \\
\text { RAM }\end{array}$ & $\begin{array}{l}\text { HOST } \\
\text { BW }\end{array}$ & $\begin{array}{l}\text { HOST } \\
\text { STORAGE }\end{array}$ \\
\hline $\begin{array}{l}\text { Power Model Spec PowerHp Pro Liant } \\
\text { M1110G4Xeon3040 }\end{array}$ & 1860 & 2 & 4096 & 1000000 & 1000000 \\
\hline $\begin{array}{l}\text { Power ModelSpec Power HpPro LiantMl } \\
\text { 110G5Xeon3075 }\end{array}$ & 2660 & 2 & 1096 & 1000000 & 1000000 \\
\hline
\end{tabular}

Table 7 Configuration of VMs

\begin{tabular}{llllll}
\hline VM TYPES & VM MIPS & VM PES & VM RAM & VM BW & VM STORAGE \\
\hline 1 & 2500 & 1 & 870 & 1000000 & 2500 \\
2 & 2000 & 1 & 870 & 1000000 & 2500 \\
3 & 1500 & 1 & 1020 & 1000000 & 2500 \\
4 & 500 & 1 & 613 & 1000000 & 2500 \\
\hline
\end{tabular}

Table 8 simulation results of performance metric

\begin{tabular}{|c|c|c|c|c|c|}
\hline Policy & $\begin{array}{l}\text { SLA } \\
\text { violations } \\
\text { (\%) }\end{array}$ & $\begin{array}{l}\text { Energy } \\
\text { consumption(Kw/Hr) }\end{array}$ & SLATAH & $\begin{array}{l}\text { PDM } \\
(\%)\end{array}$ & VM-Migration(No.) \\
\hline $\begin{array}{l}\text { ORAP with } \\
\text { Fuzzy } \lambda\end{array}$ & 3.67 & 25.7 & 7.81 & 0.22 & 4238 \\
\hline $\begin{array}{l}\text { MOOA } \\
\text { random } \lambda\end{array}$ & 5.41 & 30.2 & 9.81 & 0.26 & 4778 \\
\hline Watts per core & 10.78 & 29.8 & - & - & - \\
\hline LLC & 6.32 & 34.96 & 7.30 & 0.47 & 3345 \\
\hline $\mathrm{RR}$ & 11.10 & 30.33 & - & - & - \\
\hline NPA & - & 150.68 & - & - & - \\
\hline DVFS & - & 615.8 & - & - & - \\
\hline
\end{tabular}




\subsection{Analysis of result}

Several experiments are performed by varying the values of random co-efficient and fuzzy-based coefficient. In this section, we discuss the summarized result showing the comparison between the random $\lambda$ with fuzzy-based $\lambda$. Experiments are evaluated multiple times and summarized results are shown in Table 8. We have compared our techniques against five other existing techniques namely round robin [3], watts per core (WPC)[18], limited look ahead control (LLC) [19], non-power aware policy (NPA) [20] and dynamic voltage and frequency scaling (DVFS) [21]. The value based result comparisons are shown in Figure 2. From the results, it is analyzed that by considering variance in different characteristics of host, preference of objectives are identified clearly and subsequent impacts are visible through results. The comparisons of results are summarized in Figure 3 and 4. From the Figure 3 and 4 , we can see that ORAP with fuzzy based coefficient handles both the objectives efficiently and generates the Pareto front. The optimal solutions that give the feasible region are shown in Figure 5 subsequent discussion on the results has been made in following section.

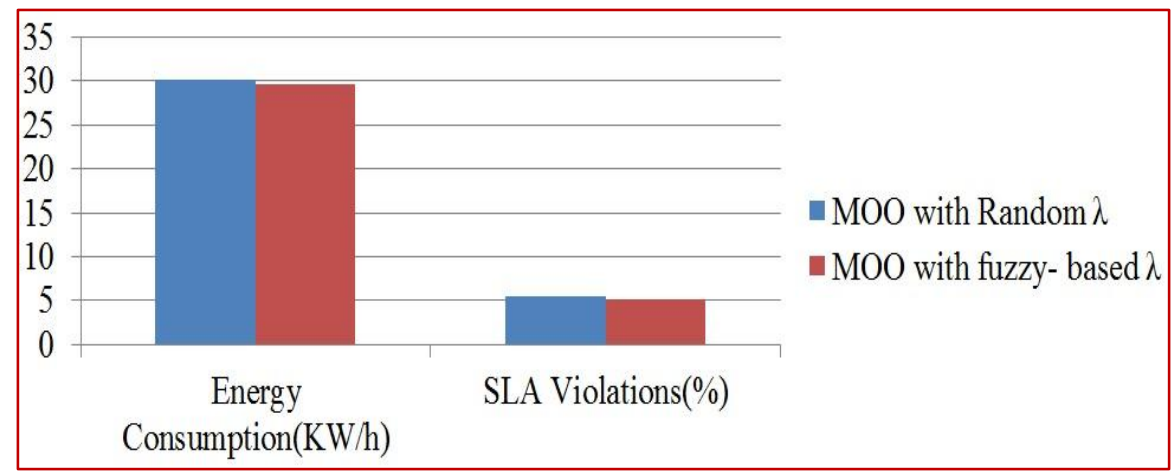

Figure 2 A comparisons of random $\lambda$ with fuzzy values of $\lambda$

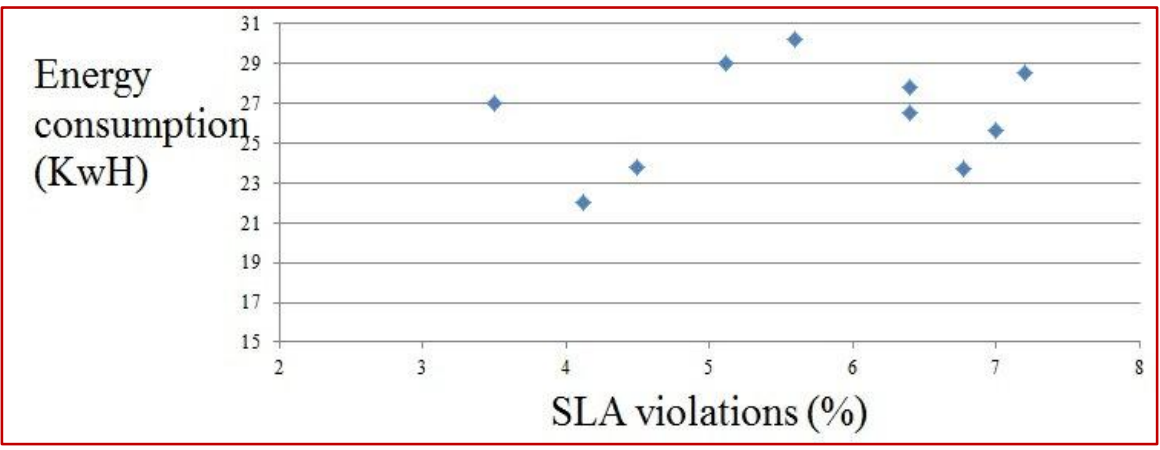

Figure 3 Average results with the random values of $\lambda$

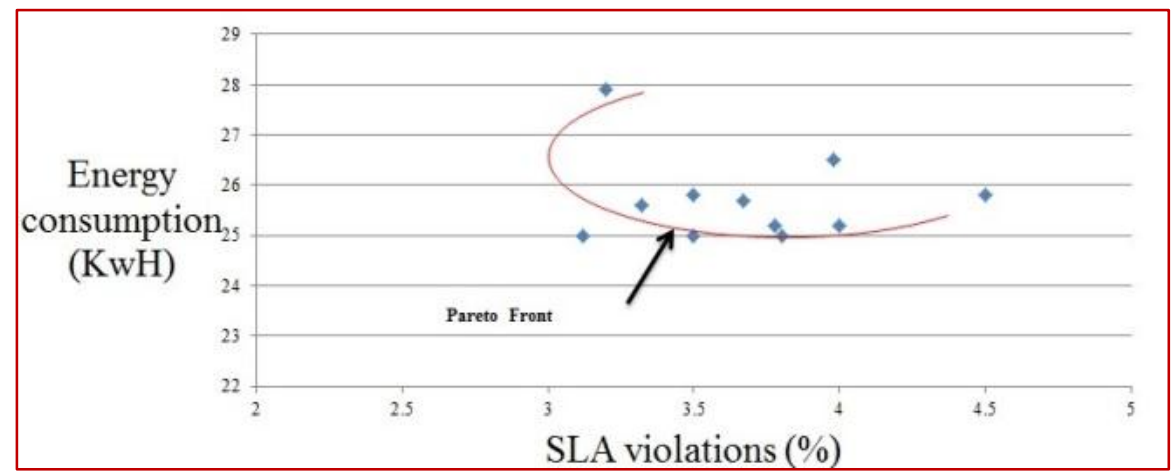

Figure 4 Average results with the fuzzy values of $\lambda$ 
Shrimali et al.

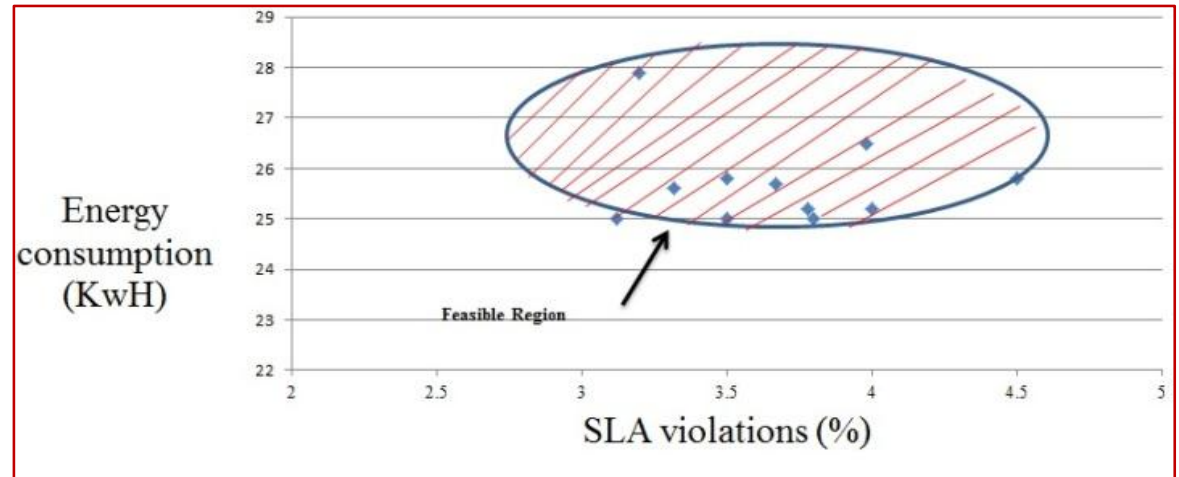

Figure 5 A feasible region generated by the fuzzy values of $\lambda$

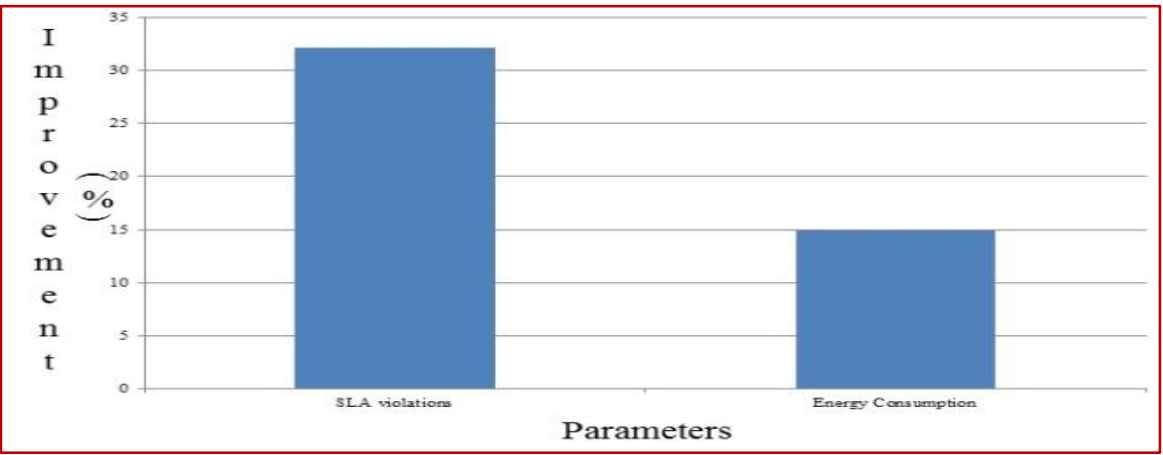

Figure 6 Improvement in energy consumption and SLA violations due to consideration of fuzzy based $\lambda$

4.3.1Impact of the co-efficient on energy efficiency and performance

Since minimizing the power consumption of data center is one of our objectives, we compare the utilization of 800 hosts included in the experiment and select the hosts with the average utilization. To identify the average utilized hosts, utilization is calculated for the period of $24 \mathrm{hrs}$. It can be noticed that while applying MOOA policy for selection of (request, host) pair with random co-efficient, it arbitrarily gives priority to objectives. Hence, when energy consumption gets more priority over performance, it predicts the possible power consumption of host and accordingly selects the most appropriate host. Energy consumption is measured in average value of $\mathrm{KW} / \mathrm{hr}$.

From the Table 8 , we can notice that using MOOA policy, power consumption is about $30.2 \mathrm{Kw} / \mathrm{hr}$. The values are nearer to optimized energy efficient technique WPC. But when fuzzy based co-efficient $\lambda$ is calculated it select the host based on its characteristics and parameters, hence it reduces the power consumption that is about $25.7 \%$. Performance is the another objective, that is targeted by minimizing the SLAV. SLAV is calculated SLAVAN and PDM. The performance of the MOOA policy quantifies by the number of SLA violations which results into the $5.41 \%$. The comparisons are shown in Table 8. It depicts that Fuzzy based MOOA technique outperforms as compared to MOOA with random co-efficient.

\section{Conclusion and future work}

We have implemented and validated a fuzzy-based approach to evaluate MOO for resource allocation in Cloud. The random co-efficient of MOO is generated without taking into considering the current status of power consumption and SLA violations of host. Fuzzy based approach generates the co-efficient based on the current status of host. Experiment evaluation using Cloudsim environment shows that an allocation using ORAP with random coefficient consumes, on average, $25.7 \%$ in power and $3.67 \%$ SLA violations over a 24-hour period with maintaining QoS goals. Also, MOOA with fuzzy coefficient depicts $32 \%$ improvement in SLAV as compared to relative risk and shows $14 \%$ reduction in power consumption as compared to random coefficient of MOO equation. Future work could be in the direction of exploring an option of incorporating MOO in various VM migration techniques. 
Acknowledgment

None.

\section{Conflicts of interest}

The authors have no conflicts of interest to declare.

\section{References}

[1] Hamilton J. Cooperative expendable micro-slice servers (CEMS): low cost, low power servers for internet-scale services. In conference on innovative data systems research (CIDR'09), 2009.

[2] Jansen R, Brenner PR. Energy efficient virtual machine allocation in the cloud. In international green computing conference and workshops 2011 (pp. 1-8). IEEE.

[3] Sempolinski P, Thain D. A comparison and critique of eucalyptus, opennebula and nimbus. In international conference on cloud computing technology and science 2010 (pp. 417-26). IEEE.

[4] Shrimali B, Patel H. Performance based energy efficient techniques for VM allocation in cloud environment. In proceedings of the third international symposium on women in computing and informatics 2015 (pp. 477-86). ACM.

[5] Marler RT, Arora JS. Survey of multi-objective optimization methods for engineering. Structural and Multidisciplinary Optimization. 2004; 26(6):369-95.

[6] Shrimali B, Patel H. Multi-objective optimization oriented policy for performance and energy efficient resource allocation in cloud environment. Journal of King Saud University-Computer and Information Sciences. 2017.

[7] Konak A, Coit DW, Smith AE. Multi-objective optimization using genetic algorithms: a tutorial. Reliability Engineering \& System Safety. 2006; 91(9):992-1007.

[8] Rao JR, Roy N. Fuzzy set theoretic approach of assigning weights to objectives in multieriteria decision making. International Journal of Systems Science. 1989; 20(8):1381-6.

[9] Masoumzadeh SS, Hlavacs H. An intelligent and adaptive threshold-based schema for energy and performance efficient dynamic VM consolidation. In European conference on energy efficiency in large scale distributed systems 2013 (pp. 85-97). Springer, Berlin, Heidelberg.

[10] Xu J, Zhao M, Fortes J, Carpenter R, Yousif M. On the use of fuzzy modeling in virtualized data center management. In international conference on autonomic computing 2007 (pp. 25-35). IEEE.

[11] Panchal R, Shrimali B, and Patel H. Entropy based method to calculate weight for multi-criterian VM allocation policy in compute cloud. International Journal of Computer Science and Mobile Applications. 2018; 6(4):170-82.
[12] Beloglazov A, Buyya R. Optimal online deterministic algorithms and adaptive heuristics for energy and performance efficient dynamic consolidation of virtual machines in cloud data centers. Concurrency and Computation: Practice and Experience. 2012; 24(13):1397-420.

[13] Lee YC, Zomaya AY. Energy efficient utilization of resources in cloud computing systems. The Journal of Supercomputing. 2012; 60(2):268-80.

[14] Jantzen J. Tutorial on fuzzy logic. Technical University of Denmark, Department of Automation, Technical Report. 1998.

[15] Mendel JM. Fuzzy logic systems for engineering: a tutorial. Proceedings of the IEEE. 1995; 83(3):345-77.

[16] Calheiros RN, Ranjan R, Beloglazov A, De Rose CA, Buyya R. CloudSim: a toolkit for modeling and simulation of cloud computing environments and evaluation of resource provisioning algorithms. Software: Practice and Experience. 2011; 41(1):23-50.

[17] Chun B, Culler D, Roscoe T, Bavier A, Peterson L, Wawrzoniak M, et al. Planetlab: an overlay testbed for broad-coverage services. ACM SIGCOMM Computer Communication Review. 2003; 33(3):3-12.

[18] Raycroft P, Jansen R, Jarus M, Brenner PR. Performance bounded energy efficient virtual machine allocation in the global cloud. Sustainable Computing: Informatics and Systems. 2014; 4(1):1-9.

[19] Kusic D, Kephart JO, Hanson JE, Kandasamy N, Jiang G. Power and performance management of virtualized computing environments via lookahead control. Cluster Computing. 2009; 12(1):1-15.

[20] Beloglazov A, Buyya R. Energy efficient allocation of virtual machines in cloud data centers. In IEEE/ACM international conference on cluster, cloud and grid computing 2010 (pp. 577-8). IEEE.

[21] Guérout T, Monteil T, Da Costa G, Calheiros RN, Buyya R, Alexandru M. Energy-aware simulation with DVFS. Simulation Modelling Practice and Theory. 2013; 39:76-91.

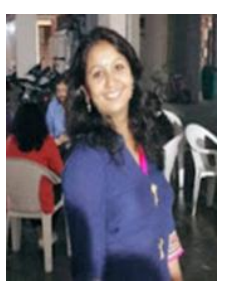

Bela Shrimali is currently working as a Assistant Professor in Computer Engineering Department of LDRPinstitute of technology and researchGandhinagar, Gujarat, India. She completed her masters in computer science and engineering from Government engineering college, Gandhinagar-India and completed her under graduation (BE-Computer Engineering) from Gujarat University, Ahmedabad-India. She is having more than 12 years of teaching experience. Ms. Shrimali has presented and published many research papers in various conferences \& journals of international/national repute. Currently, she is pursuing $\mathrm{PhD}$ in the domain of Cloud Computing with resource allocation as specialization.

Email: Bela.shrimali@gmail.com 
Shrimali et al.

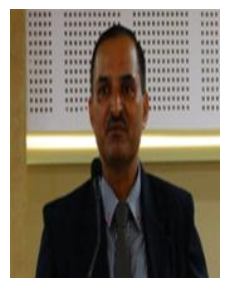

Dr. H B. Bhadka is currently working as a Dean at faculty of Computer Science, C. U. Shah University, Wadhwan City. Gujarat. India. He completed his $\mathrm{PhD}$ from Saurashtra University Rajkot in june2009. He has having more than 14 years of teaching experience. Dr. Bhadka has many research papers in various conferences \& journals of international repute. He has published more than 10 books and delivered many expert talks at different universities. He has attended many workshops, STTP and training programmes. Dr. Bhadka has also evaluated more than seven $\mathrm{PhD}$ theses.

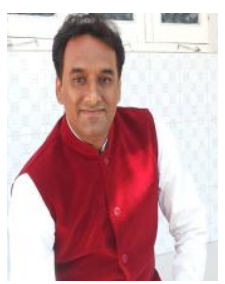

Dr. Hiren B. Patel is currently working as a Professor and Head in Computer Engineering Department of LDRP-institute of technology and research-Gandhinagar, Gujarat, India. He completed his Ph.D. from National Institute of Technology (NIT), Surat with Cloud computing as the domain of research. He completed his post graduation from M. S. University, Baroda and graduation from S. P. University, V.V.Nagar. Having more than 15 years of teaching experience. Dr. Patel has many research papers in various conferences \& journals of international repute. Few of his main subjects of interest are Computer Programming, Cloud computing, Parallel Processing, Computer Networking and Information Security. 